\title{
Nanostructuring and oxidation of diamond by two-photon ultraviolet surface excitation: An XPS and NEXAFS study
}

\author{
C. G. Baldwin, ' J. E. Downes, C. J. McMahon, C. Bradac, and R. P. Mildren \\ MQ Photonics Research Centre, Department of Physics and Astronomy, Macquarie University, New South Wales 2109, Australia ${ }^{\dagger}$
}

(Received 7 January 2014; revised manuscript received 15 April 2014; published 15 May 2014)

\begin{abstract}
We report $\mathrm{C}(1 s)$ and $\mathrm{O}(1 s)$ surface sensitive $\mathrm{x}$-ray photoelectron spectroscopy (XPS) and $\mathrm{C}$ and $\mathrm{O} K$-edge partial-electron yield near-edge $\mathrm{x}$-ray absorption fine structure (NEXAFS) measurements for (100) and (110) oxidized diamond surfaces, etched by a laser two-photon ultraviolet (UV) desorption process. Etched regions of the (100) surface show increased oxygen coverage with a higher fraction of singly bonded termination species than unetched regions. Similar changes are observed for the (110) but with smaller magnitude. For both surfaces, no major change in $s p^{2}$ bonded carbon is observed. We show that the terminations observed for etched surfaces are consistent with the formation of oxidized $\{111\}$ facets. For deeply etched samples, atomic force microscopy and scanning electron microscopy confirm the presence of $\{111\}$-like facets and reveals the development of nanoscale facetted ridges directed perpendicular to the etching beam polarization. An etching mechanism is proposed involving localized optical absorption by surface electronic states, with the probability for subsequent desorption events varying according to the relative directions of laser polarization and lattice orientation.
\end{abstract}

DOI: 10.1103/PhysRevB.89.195422

PACS number(s): 81.05.ug, 68.43.Tj, 79.60.-i, 81.16.Rf

\section{INTRODUCTION}

Diamond is a wide band gap semiconductor which possesses properties of intense interest in many fields of science and technology [1-3]. Progress in many research directions is heavily reliant upon a limited number of techniques used to structure, shape, and polish diamond devices. This need is particularly acute in the development of resonant optical cavities and waveguides, incorporating carefully positioned color centers for applications in quantum computing and nanoscale magnetometry [4-6]. The current techniques for processing diamond are based on mechanical polishing, laser ablation, plasma etching, and ion implantation. However, creating structures with the fidelity achieved in other semiconductor materials is notoriously difficult because of diamonds extreme strength, hardness, and resistance to chemical attack. Direct two-photon desorption of carbon from diamond [7] is a promising laser direct-write approach for the creation of high resolution, graphite free, structures that enable an enhanced range of application capabilities.

Two-photon desorption, also referred to as nanoablation, occurs at optical fluences below the threshold for conventional laser ablation, and has been observed for laser photon energies just below the band gap energy of diamond. Kononenko et al. [8] first observed that pulsed UV $(248 \mathrm{~nm})$ irradiation of single crystal diamond surfaces in air at subablation pulse fluences resulted in the slow removal of the surface at a rate on the order of $1 \mathrm{pm} /$ pulse. Using $266 \mathrm{~nm}$ light on high grade single crystal diamond, Mildren et al. [7] found that the etch rate follows a quadratic dependence with pulse fluence. This quadratic dependence was also observed across the beam profile, showing that the nonlinear nature of the process can be exploited to produce features smaller than the diffraction

\footnotetext{
*Corresponding author: christopher.baldwin@students.mq.edu.au

†http://web.science.mq.edu.au/groups/diamond/
}

limited size of the beam. As intensity was reduced, no threshold was obtained with etch rates as low as $3 \times 10^{-9} \mathrm{~nm}$ per pulse (corresponding to several tens of atoms per pulse) so far demonstrated using nanojoule UV pulses [9]. Such an etch rate is many orders of magnitude lower than the minimum obtained using laser ablation (typically $1 \mathrm{~nm} /$ pulse). At high fluences, controlled etch rates up to $10 \mathrm{pm} /$ pulse (corresponding to much less than an atomic layer per pulse) are achievable using nanosecond pulses providing the ablation threshold is not exceeded. The presence of atmospheric oxygen is necessary [8], indicating that the process is photochemical in nature and sustained by surface oxidation. Initial near edge $\mathrm{x}$-ray absorption fine structure (NEXAFS) measurements revealed that the etched surface is oxygen terminated and graphite free. A lack of detritus on the sample suggests the etching by-products are highly volatile species such as $\mathrm{CO}$ and $\mathrm{CO}_{2}$.

These characteristics of material removal, which to the authors knowledge are distinctive to diamond, have led to a broad description of a "cold" etch mechanism where twophoton absorption occurs in the diamond, as opposed to the surrounding gas, which results in the ejection of carbon and oxygen atoms followed by readsorption of oxygen from the atmosphere to regenerate the oxygen termination layer. However, many aspects of the mechanism are poorly understood. Important characteristics that determine minimum feature size, as well as the smoothness and chemical characteristics of etched surfaces, are yet to be elucidated.

In the present work, we analyze the atomic populations and bonding characteristics of etched diamond surfaces in detail, with a view to understanding the specific photochemical mechanism responsible for the etching process. We used surface sensitive XPS and NEXAFS to characterize oxygen-terminated (100) and (110) surfaces upon two-photon etching. XPS allows the measurement of oxygen species on the surface, both directly as well as indirectly via the fine structure of carbon photoelectron spectra, making it well suited 
to the identification of distinct termination species. Some species, such as double bonded oxygen, produce electronic states in the band gap which are directly observable via NEXAFS [10-12].

The chemical environment of a diamond surface is complex and has been found to depend strongly on the crystal facet orientation and the method of surface preparation. The total amount of oxygen on a fully oxidized diamond surface varies with the type of crystallographic face-(100) surfaces saturate at $0.5 \mathrm{ML}$, while (110) and (111) faces saturate at 0.33 ML [13-15]. The (100) surface supports a mixture of $\mathrm{C}-\mathrm{O}-\mathrm{C}$ (ether), $\mathrm{C}-\mathrm{OH}$ (hydroxyl), and $\mathrm{C}=\mathrm{O}$ (ketone) groups at lower coverages, that becomes dominated by ethers as the coverage increases [16-18]. The bias towards ether and hydroxyl groups over ketones is also supported theoretically [19]. The (110) face has been reported to be populated exclusively by ethers [20] or a mixture of ether and ketone groups [15,21]. These reports investigated (110) surfaces prepared by various combinations of mechanical polishing and hydrogen plasma treatment, both of which tend to result in a surface containing $\{111\}$ microfacets and step edges, rather than atomically flat (110) terraces [22]. It is likely that the varied findings reflect the range of conditions that may be present on a nominally (110) surface. (111) faces contain primarily ether groups, with the $(2 \times 1)$ reconstruction being the most energetically favorable [15,18,23,24]. Brobov et al. [20] report different results, finding also ketone groups on (111) diamond oxidized by room temperature exposure to molecular oxygen.

In the light of this knowledge of surface compositions, $\mathrm{X}$-ray measurements of species populations are used to quantify surface changes induced by two-photon UV etching. In the first section we describe the procedure for two-photon etching and subsequent $\mathrm{X}$-ray surface analysis. The following sections then analyze populations as determined by XPS and NEXAFS, and changes in surface texture as measured by scanning electron microscopy (SEM) and atomic force microscopy (AFM). We then discuss the implications of these results for the etching mechanism and by extension for applications of the technique in diamond surface processing.

\section{EXPERIMENTS}

A rectangular prism of type IIa CVD-grown single crystal diamond was cut to expose (100) and (110) facets and mechanically polished to $1 \mathrm{~nm}$ roughness (Element 6). Prior to etching, the sample was prepared by cleaning in piranha solution at $120^{\circ} \mathrm{C}$ to ensure an oxygen terminated surface [25]. Rectangular etched regions were then prepared on the (100) and (110) crystal faces by raster scanning the focus of a pulsed $266 \mathrm{~nm}$ laser beam across the surface. The laser was a $Q$-switched fourth harmonic Nd:YAG system producing $10 \mathrm{~ns}$ pulses with a $10 \mu \mathrm{m}$ beam waist and energy of $12.5 \mu \mathrm{J} /$ pulse with a $14 \mathrm{kHz}$ repetition rate. The pulse fluence was $16 \mathrm{~J} / \mathrm{cm}^{2}$, well below the ablation threshold of $100 \mathrm{~J} / \mathrm{cm}^{2}$ [7] (defined here as the minimum fluence at which there is a $50 \%$ chance of ablation for a burst of $10^{3}$ pulses). A feed rate of $1 \mathrm{~mm} / \mathrm{min}$ was chosen to provide adequate depth from a single pass with minimal risk of ablation. The (100) sample (Fig. 1) was etched to a depth of $(9 \pm 1) \mathrm{nm}$ over a $600 \times 200 \mu \mathrm{m}^{2}$ area, while

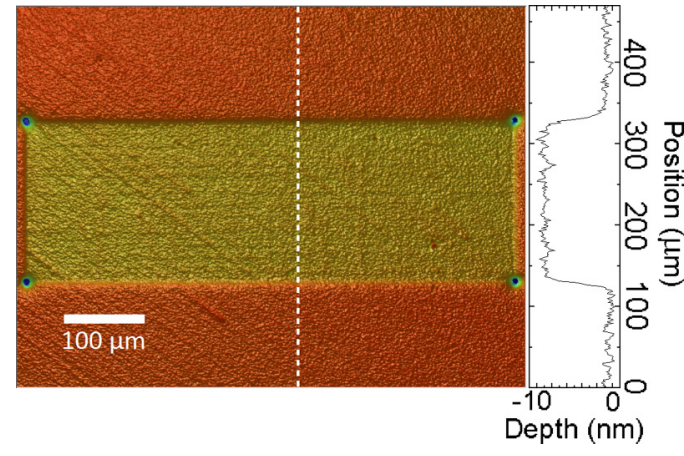

FIG. 1. (Color online) Optical profiler image of the etched (100) surface. The etched region measures $600 \times 200 \mu \mathrm{m}^{2}$ and is $(9 \pm 1) \mathrm{nm}$ deep. In order to assist location of the etched region inside the x-ray analysis chamber, deep pits of depth approximately several hundred nanometres were etched at the corners by stationary exposure of the etching beam for $1 \mathrm{~min}$.

the (110) sample was etched to $(7 \pm 2) \mathrm{nm}$ over a $1000 \times$ $200 \mu \mathrm{m}^{2}$ area.

The prepared samples were analyzed using the soft X-ray beamline at the Australian Synchrotron. XPS and NEXAFS measurements were performed under ultrahigh vacuum (UHV) conditions with background pressure below $10^{-10}$ mbar. The source was an Apple II elliptically polarizing undulator, which allowed arbitrary selection of beam polarization. The focused beam was incident at $45^{\circ}$ from the sample normal to form a $150 \times 500 \mu \mathrm{m}^{2}$ spot on the diamond surface. The PHOIBOS 150 hemispherical analyzer was positioned normal to the sample. A flood gun was used (electron energy $2 \mathrm{eV}$ ) to ameliorate charging effects.

The sample was ultrasonically cleaned in acetone prior to insertion into the beamline. After insertion, the sample was heated to $550{ }^{\circ} \mathrm{C}$ in situ under UHV conditions. This temperature was chosen in order to remove any physisorbed contaminants without causing the thermal desorption of chemisorbed oxygen [20,21]. Preliminary XPS survey scans were performed with a photon energy of $1486.7 \mathrm{eV}$ (corresponding to the Al $K \alpha_{1}$ transition). These confirmed that the samples contained only carbon, oxygen, and trace amounts of silicon. ${ }^{1}$ Greater surface sensitivity was achieved by using photon energies of $395 \mathrm{eV}$ and $640 \mathrm{eV}$ to measure the $\mathrm{C}(1 s)$ and $\mathrm{O}(1 s)$ states, respectively. These energies ensure that the kinetic energy of electrons from the feature of interest is approximately $100 \mathrm{eV}$, where the electron mean free path in the diamond bulk is a minimum [26]. Measurements were performed at the center of the etched region and for a nearby unetched reference region.

XPS spectra were deconvolved using XPSPEAK4.1 software, applying a Shirley background subtraction. As type IIa diamond is an excellent electrical insulator, XPS measurements

\footnotetext{
${ }^{1}$ Weak photoelectron peaks were observed at approximately $150 \mathrm{eV}$ and $100 \mathrm{eV}$, in the region of the silicon $2 s$ and $2 p$ states, respectively. We attribute these peaks to silica impurities originating from the glass beaker used to clean the sample. The intensity is negligible compared to the total oxygen signal.
} 
are susceptible to the effects of surface charging. Despite the use of a flood gun, rigid shifts in core level binding energies of the order $1 \mathrm{eV}$ were observed, making extraction of absolute binding energy values unreliable. Assignment of the observed components of the XPS spectra was therefore deduced using the relative splittings of subpeaks in the deconvolved spectra.

NEXAFS measurements were performed in Auger electron yield (AEY) mode in order to obtain the sensitivity necessary to observe surface states in the band gap. The electron analyzer window was centered at $234 \mathrm{eV}$ and $100 \mathrm{eV}$ for carbon and oxygen $K$ edges, respectively. The data obtained was double normalized to remove the effects of beam flux changes. The beam flux was obtained using a gold mesh placed in the x-ray beam. Carbon contamination present on the beamline optical surfaces results in a distortion of the mesh signal. To account for this, a set of reference scans were performed on a gold surface cleaned by argon ion sputtering and used to normalize beam flux measurements. Measurements were performed with $\mathrm{x}$-ray beam in $p$ and $s$ polarizations in order to gain further information on average bond directions [27]. For the (100) surface, $p$ and $s$ polarizations were parallel to the [110] and [010] directions, respectively, while for the (110) surface, $p$ and $s$ polarizations were parallel to the [100] and [001] directions.

Scanning electron microscopy (SEM) and atomic force microscopy (AFM) were used to investigate the structure of deeply etched (100) and (110) samples (depth $\sim 500 \mathrm{~nm}$ and $\sim 350 \mathrm{~nm}$, respectively). Images were obtained using a field-emission SEM (JEOL JSM-7800F). Surface profiles were obtained by AFM (MT-NDT Ntegra Spectra) in noncontact mode using a silicon tip, with a radius of curvature of $10 \mathrm{~nm}$ (NT-MDT NSG01). The effects of the finite tip radius were partially removed by image deconvolution using image analysis software (Image Analysis 3, NT-MDT).

\section{RESULTS}

\section{A. XPS C(1s)}

C(1s) XPS spectra consist of an intense central peak attributed to $s p^{3}$ bonded bulk diamond, with subpeaks arising from surface carbon atoms terminated by oxygen at higher energies, and a lower binding energy subpeak due to $s p^{2}$ bonded carbon (Fig. 2). Comparison of the separations of subpeak binding energies from the main $s p^{3}$ peak with results obtained in other reports $[14,18,24,28-30]$ allowed the attribution of the higher binding energy subpeaks to single and double oxygen bonded carbon. The double bonded oxygen subpeak is assumed to be primarily due to ketone $(\mathrm{C}=\mathrm{O})$ groups, while the single bonded carbon subpeak may be due to either ether $(\mathrm{C}-\mathrm{O}-\mathrm{C})$, hydroxyl $(\mathrm{C}-\mathrm{OH})$, or a mixture of both. The (100) measurements also show a wide, low area, peak with low binding energy present on the surface that is diminished on the etched surface. Although the energy location may suggest it is due to $s p$ bonded carbon, we have been unable to conclusively identify the origin of this peak.

A quantitative comparison of etched and unetched spectra in Table I reveals a number of significant changes in the surface

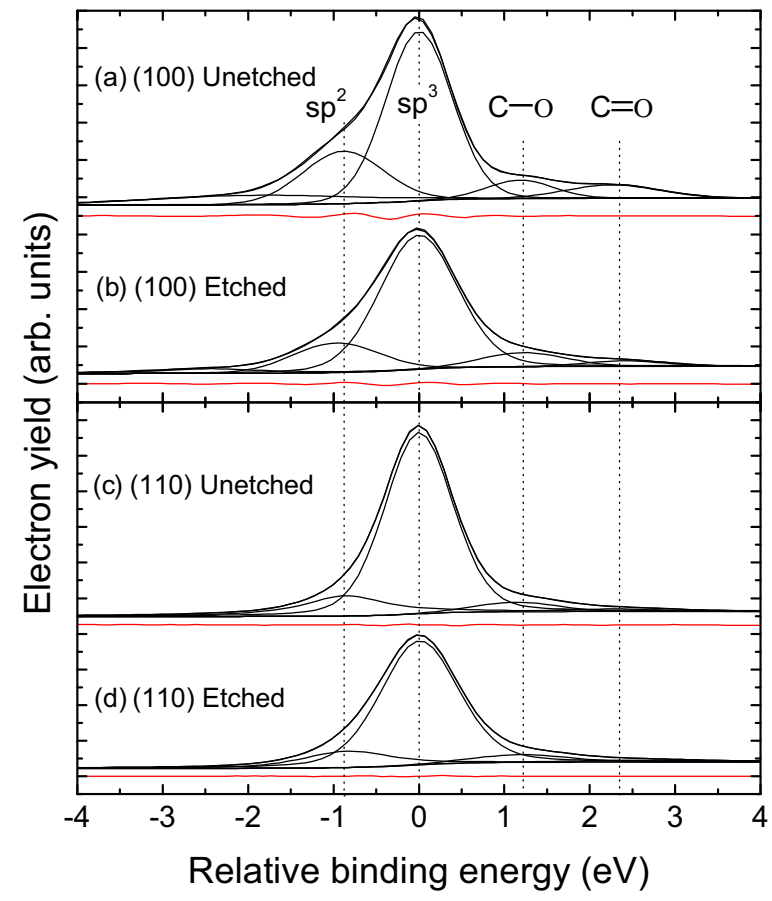

FIG. 2. (Color online) Deconvolved C(1s) XPS spectra at $395 \mathrm{eV}$ photon energy. The residuals are shown in red under the appropriate spectra. The spectra for each surface were obtained at a constant beam flux to enable quantitative comparison of surface species.

environment. For both the (100) and (110) surfaces, there is a reduction in the $s p^{2}$ peak: approximately $58 \%$ for the (100) and approximately $25 \%$ for the (110). On the (100) surface there is a substantial reduction in doubly bonded signals upon etching, indicating a significantly altered chemical environment. The (110) face shows largely unchanged oxygen related signals.

We note that lower total integrated $\mathrm{C}(1 s)$ signals are systematically measured from the etched surfaces. This is evident in both the $395 \mathrm{eV}$ and $1486.7 \mathrm{eV}$ photon energy measurements, where the integrated signal is $22 \%$ and $12 \%$ lower, respectively, on the (100) etched surface and $22 \%$ and $8 \%$ lower on the (110) surface. The beam flux did not significantly change between measurements performed on the same face, so the lower intensity is attributed to altered conditions on the surface. Since it is more pronounced in the more surface sensitive $395 \mathrm{eV}$ measurements, we infer that it is caused by the change in surface texture induced by etching (as discussed below) which may alter the sensitivity factors for photoelectrons generated from subsurface carbon. In particular, increased roughness results in a lower signal due to a broadening of the angular distribution of photoelectrons [31].

\section{B. XPS O(1s)}

A comparison of $\mathrm{O}(1 s)$ XPS spectra for etched and unetched surfaces is shown in Fig. 3. Examining the deconvolved subpeaks, it can be seen that the spectra are dominated by a large, higher binding energy peak, attributed primarily to ether groups and a smaller peak approximately $2 \mathrm{eV}$ lower attributed 
TABLE I. Binding energies (B.E.) and areas of subpeaks from deconvolved C(1s) $395 \mathrm{eV}$ XPS spectra. Binding energies are given relative to the position of the dominant $s p^{3}$ peak. As measurements for a given face were performed with the same beam flux and sample geometry, direct area comparisons can be made between the two areas of the same face. The uncertainty in peak locations and areas correspond to estimates based on manual iteration of parameters that produce a negligible residual trace.

\begin{tabular}{|c|c|c|c|c|c|c|c|}
\hline \multirow[b]{3}{*}{ Face } & \multirow[b]{3}{*}{ Species } & \multirow{2}{*}{\multicolumn{3}{|c|}{$\begin{array}{l}\mathrm{C}(1 s) \text { XPS subpeaks } \\
\text { Unetched surface }\end{array}$}} & \multirow{2}{*}{\multicolumn{3}{|c|}{ Etched surface }} \\
\hline & & & & & & & \\
\hline & & $\begin{array}{l}\Delta \mathrm{B} . \mathrm{E} . \\
(\mathrm{eV})\end{array}$ & $\begin{array}{c}\text { Subpeak area } \\
\left(10^{4} \text { counts }\right)\end{array}$ & $\begin{array}{c}\text { Area fraction } \\
(\%)\end{array}$ & $\begin{array}{l}\Delta \text { B.E. } \\
(\mathrm{eV})\end{array}$ & $\begin{array}{c}\text { Subpeak area } \\
\left(10^{4} \text { counts }\right)\end{array}$ & $\begin{array}{c}\text { Area fraction } \\
(\%)\end{array}$ \\
\hline \multirow[t]{6}{*}{ (100) } & Unknown & $-1.8 \pm 0.5$ & $16 \pm 4$ & $10 \pm 3$ & $-2.4 \pm 0.5$ & $5.9 \pm 1$ & $5 \pm 1$ \\
\hline & $s p^{2}$ & $-0.9 \pm 0.2$ & $34 \pm 10$ & $21 \pm 6$ & $-1.0 \pm 0.2$ & $20 \pm 7$ & $16 \pm 6$ \\
\hline & $s p^{3}$ & 0 & $91 \pm 10$ & $57 \pm 6$ & 0 & $87 \pm 10$ & $69 \pm 8$ \\
\hline & $\mathrm{C}-\mathrm{O}$ & $+1.2 \pm 0.1$ & $9.7 \pm 0.5$ & $6.0 \pm 0.3$ & $+1.2 \pm 0.1$ & $9.3 \pm 0.5$ & $7.4 \pm 0.4$ \\
\hline & $\mathrm{C}=\mathrm{O}$ & $+2.3 \pm 0.1$ & $9.7 \pm 0.5$ & $6.0 \pm 0.3$ & $+2.4 \pm 0.1$ & $3.3 \pm 0.2$ & $2.6 \pm 0.2$ \\
\hline & Total & & 160.5 & & & 125.8 & \\
\hline \multirow[t]{5}{*}{ (110) } & $s p^{2}$ & $-0.8 \pm 0.2$ & $20 \pm 5$ & $14 \pm 4$ & $-0.8 \pm 0.2$ & $15 \pm 5$ & $13 \pm 5$ \\
\hline & $s p^{3}$ & 0 & $110 \pm 5$ & $79 \pm 4$ & 0 & $89 \pm 5$ & $79 \pm 5$ \\
\hline & $\mathrm{C}-\mathrm{O}$ & $+1.1 \pm 0.1$ & $6.5 \pm 0.5$ & $4.7 \pm 0.4$ & $+1.2 \pm 0.1$ & $6.1 \pm 0.5$ & $5.4 \pm 0.4$ \\
\hline & $\mathrm{C}=\mathrm{O}$ & $+2.2 \pm 0.1$ & $2.3 \pm 0.2$ & $1.7 \pm 0.2$ & $+2.5 \pm 0.1$ & $2.2 \pm 0.2$ & $2.0 \pm 0.2$ \\
\hline & Total & & 140.1 & & & 109.9 & \\
\hline
\end{tabular}

to ketone groups [14]. For the unetched (100) surface, the FWHM of the ketone peak is greater than that observed in other spectra, and its separation from the singly bonded oxygen

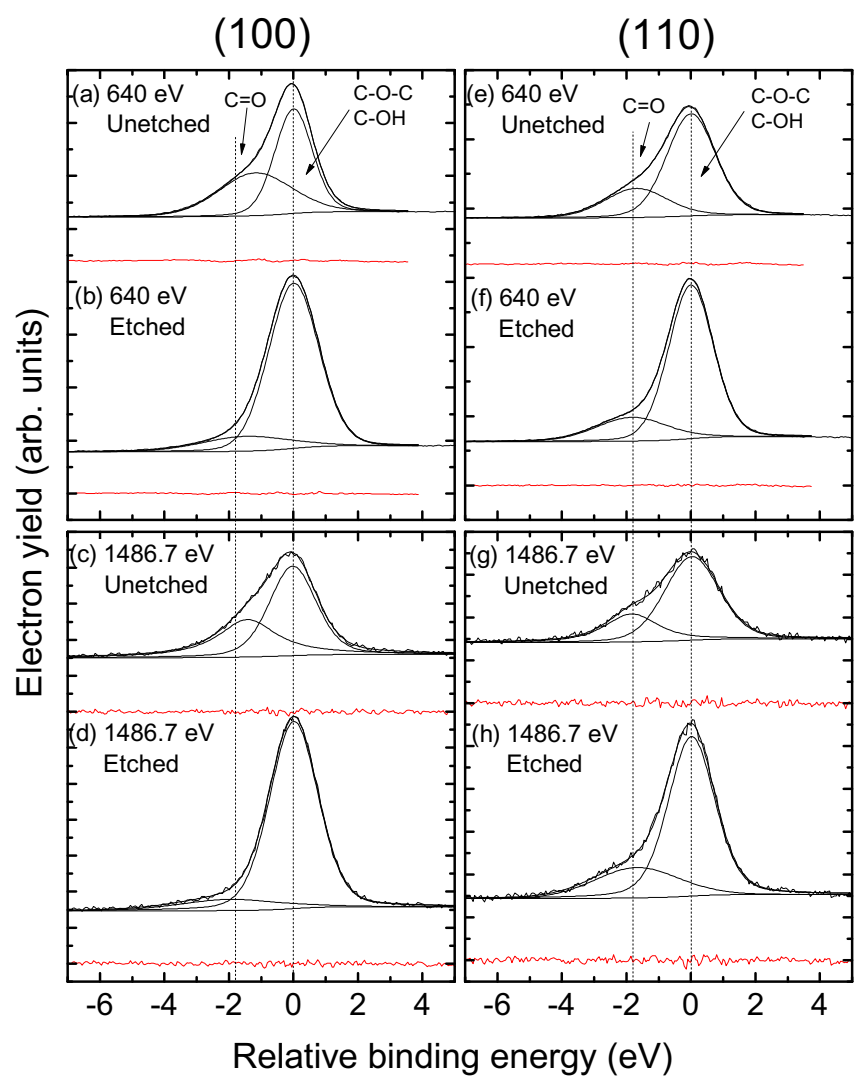

FIG. 3. (Color online) Deconvolved O $(1 s)$ XPS spectra at $640 \mathrm{eV}$ and $1486.7 \mathrm{eV}$ photon energy. Residuals are displayed in red under the appropriate spectra. Measurements are directly comparable only between measurements on the same surface at the same photon energy. peak is anomalously small. As the sample was wet oxidized in piranha solution and (100) surfaces are known to support $\mathrm{C}-\mathrm{OH}$ groups at lower coverages, it is likely that there are hydroxyl groups present on the unetched surface. However, there are few reported results for $\mathrm{O}(1 s)$ XPS binding energies for diamond surfaces in the literature and amongst these results there is some disagreement in the identification of subpeaks. The authors are aware of only two O( $1 s)$ XPS studies of single crystal diamond: Makau and Derry [14] reported binding energies of $531.5 \mathrm{eV}, 532.2 \mathrm{eV}$, and $533.1 \mathrm{eV}$ for ketone, hydroxyl, and ether groups, respectively, while Petrick et al. reported $532.5 \mathrm{eV}$ for ketone and $532.3 \mathrm{eV}$ for hydroxyl, and no ether energy was identified. Due to the apparent close spacing of hydroxyl and ether peaks and the uncertainty in their locations, we were unable to deconvolve these two groups.

$\mathrm{O}(1 s)$ subpeak areas and energies are shown in Table II. For both (100) and (110) surfaces, etching induces a significant increase in the total oxygen signal and a reduction in the fraction of ketone groups. The changes are greatest on the (100) surface where the oxygen signal increases by $32 \%$ and the fraction of ketone reduces from $43 \%$ to $13 \%$. On the (110) surface, the oxygen increase is $24 \%$ and the ketone fraction decreases from $27 \%$ to $20 \%$. The decreases in ketone are consistent (within the uncertainties of curve fitting) with the values obtained from the $\mathrm{C}(1 s)$ measurements above [from $50 \%$ to $35 \%$ for (100) and from $26 \%$ to $27 \%$ for (110)], and indicate that the etched surface either preferentially supports singly bonded groups, or the etching process directly alters the surface species by preferentially removing ketone groups.

\section{NEXAFS C $(1 s)$}

Figure 4 shows the normalized carbon $K$-edge NEXAFS spectra. The $\mathrm{C}(1 s)$ core-hole exciton is clearly visible as the sharp peak at $289.2 \mathrm{eV}$. Here we focus on the preedge features at $285.0 \mathrm{eV}$ and $286.7 \mathrm{eV}$ observed on each surface for both $p$ and $s$ polarized x-ray beams. The $285.0 \mathrm{eV}$ peak is attributed to the $\mathrm{C}(1 s)-\pi *$ resonance, associated with $s p^{2}$ 
TABLE II. Binding energies (B.E.) and integrated areas of subpeaks from deconvolved $\mathrm{O}(1 s) 640 \mathrm{eV}$ XPS spectra. Binding energies are given relative to the position of the singly bonded peak. As in Table I, direct area comparisons can be made between two areas on the same face and the quoted uncertainties are based on estimates based on manual iteration of parameters that produce a negligible residual trace.

\begin{tabular}{|c|c|c|c|c|c|c|c|}
\hline \multirow[b]{3}{*}{ Face } & \multirow[b]{3}{*}{ Species } & \multirow{2}{*}{\multicolumn{3}{|c|}{$\begin{array}{l}\mathrm{O}(1 s) \text { XPS subpeaks } \\
\text { Unetched surface }\end{array}$}} & \multirow{2}{*}{\multicolumn{3}{|c|}{ Etched surface }} \\
\hline & & & & & & & \\
\hline & & $\begin{array}{l}\Delta \mathrm{B} . \mathrm{E} . \\
(\mathrm{eV})\end{array}$ & $\begin{array}{c}\text { Subpeak area } \\
\left(10^{4} \text { counts }\right)\end{array}$ & $\begin{array}{c}\text { Area fraction } \\
(\%)\end{array}$ & $\begin{array}{c}\Delta \mathrm{B} . \mathrm{E} . \\
(\mathrm{eV})\end{array}$ & $\begin{array}{c}\text { Subpeak area } \\
\left(10^{4} \text { counts }\right)\end{array}$ & $\begin{array}{c}\text { Area fraction } \\
(\%)\end{array}$ \\
\hline \multirow[t]{3}{*}{$(100)$} & $\mathrm{C}=\mathrm{O}$ & $-1.2 \pm 0.2$ & $12 \pm 2$ & $43 \pm 7$ & $-1.8 \pm 0.2$ & $5 \pm 2$ & $13 \pm 5$ \\
\hline & $\mathrm{C}-\mathrm{O}$ & 0 & $16 \pm 2$ & $57 \pm 7$ & 0 & $32 \pm 2$ & $87 \pm 5$ \\
\hline & Total & & 27.9 & & & 36.7 & \\
\hline \multirow[t]{3}{*}{ (110) } & $\mathrm{C}=\mathrm{O}$ & $-1.7 \pm 0.2$ & $5.5 \pm 0.7$ & $27 \pm 3$ & $-1.8 \pm 0.2$ & $5.0 \pm 1$ & $20 \pm 4$ \\
\hline & $\mathrm{C}-\mathrm{O}$ & 0 & $14.9 \pm 0.7$ & $73 \pm 3$ & 0 & $20 \pm 1$ & $80 \pm 4$ \\
\hline & Total & & 20.5 & & & 25.4 & \\
\hline
\end{tabular}

bonded carbon, and the smaller $286.7 \mathrm{eV}$ peak to the $\mathrm{C}=\mathrm{O}$ $\mathrm{C}(1 s)-\pi *$ resonance $[11,12,32]$.

The $\mathrm{C}=\mathrm{O} \pi^{*}$ resonance is observed as a weak peak on a background. It is more pronounced on the (100) surface, which was shown by the XPS measurements to have a significant population of ketone groups. In agreement with XPS results, the ketone peak is smaller relative to the background on the etched compared to unetched (100) surfaces, while little change is observed on the (110) surfaces. A larger peak is observed with an $s$ polarized x-ray beam. As we expect maximum coupling into $\pi^{*}$ states when the beam polarization is oriented perpendicularly to the bond direction, the average $\mathrm{C}=\mathrm{O}$ bond direction is deduced to have a larger component

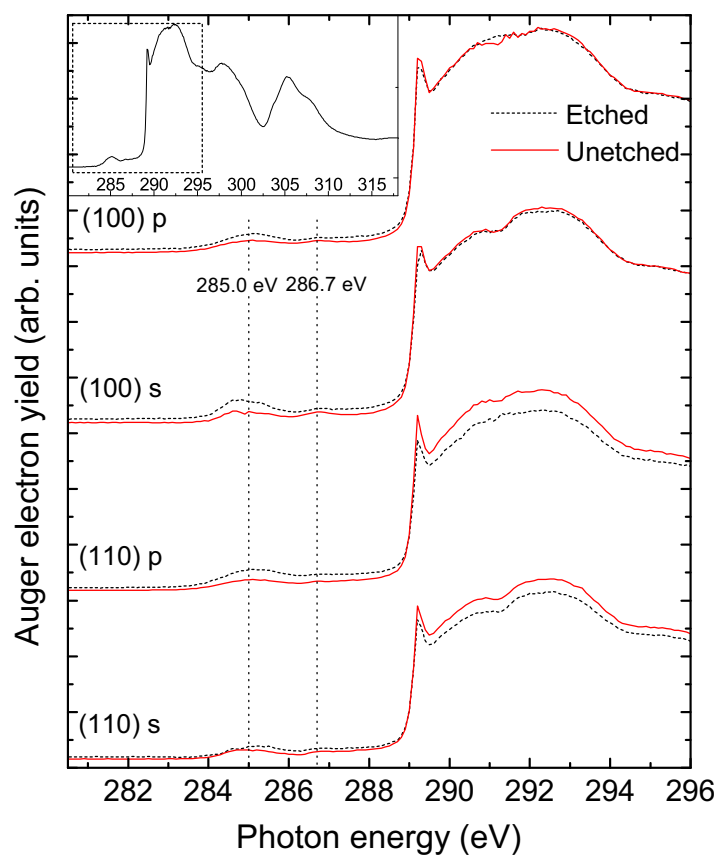

FIG. 4. (Color online) Preedge regions of normalized carbon $K$-edge AEY NEXAFS for (100) and (110) oriented surfaces with $p$ and $s$ polarized $\mathrm{x}$ rays incident on the surface at $45^{\circ}$. Features at $285.0 \mathrm{eV}$ and $286.7 \mathrm{eV}$ are attributed to the $\mathrm{C}(1 s)-\pi *$ resonances due to $\mathrm{C}=\mathrm{C}$ dimers and $\mathrm{C}=\mathrm{O}$ bonds, respectively. The inset shows a wider view of a typical spectrum, with the second band gap of diamond visible at $302.4 \mathrm{eV}$. normal to the plane of the surface, as expected for the (100) surface structure.

The $285.0 \mathrm{eV}$ feature attributed to $s p^{2}$ was stronger on etched surfaces in all spectra, in contrast to the above $\mathrm{C}(1 s)$ XPS results, which show systematic decrease in $s p^{2}$ signal. To explain this discrepancy, we note that the XPS and NEXAFS are not necessarily sampling the same atomic populations. XPS relies on measuring unscattered electrons, whereas AEY NEXAFS detects scattered electrons. As the signal weighting as a function of depth will be different for the two techniques, the sensitivity will depend on surface geometry factors that result from any changes in the surface morphology induced by the etching. Although we are unable to quantitatively explain the discrepancy, it is clear from both the XPS and NEXAFS measurements that the etching process does not result in a significant increase in $s p^{2}$ carbon on the surface.

It is also interesting to note that we observe a change in the directionality of the $s p^{2}$ signal upon etching, as determined by the polarization dependence. In contrast to the unetched surfaces, the etched surfaces exhibit a significant change in the $285 \mathrm{eV}$ peak with beam polarization. For the (100) face, the $s$ polarized signal is enhanced, while the converse is observed for the (110) face. Thus the orientation of $s p^{2}$ character bonding orbitals appear to be more out of plane for the (100) etched surface and more in plane for (110). The relevant $\mathrm{C}=\mathrm{C}$ bonds may be formed either as isolated dimers on the surface, representing individual defects with a collective average orientation, or as long rows of dimers brought about by surface reconstruction (Pandey chains) on the $\{111\}$ microfacets exposed by etching (as detailed below).

\section{NEXAFS O(1s)}

Oxygen $K$-edge NEXAFS results are shown in Fig. 5. As the measurements probed only a fraction of a monolayer of oxygen atoms, the signal was very weak. A sloping background signal is present in these spectra, which is attributed to weak instrumentation effects that persist through the normalization process described above and become significant under these low-signal conditions.

We used the previous work of Reinke et al. [33] and Gao et al. [34] to interpret the spectra. Two distinct peaks are observed which we have labeled A and B (observed at $531.9 \mathrm{eV}$ and $538.0 \mathrm{eV}$ ). Peak B is attributed to the $\sigma^{*}$ resonance and 


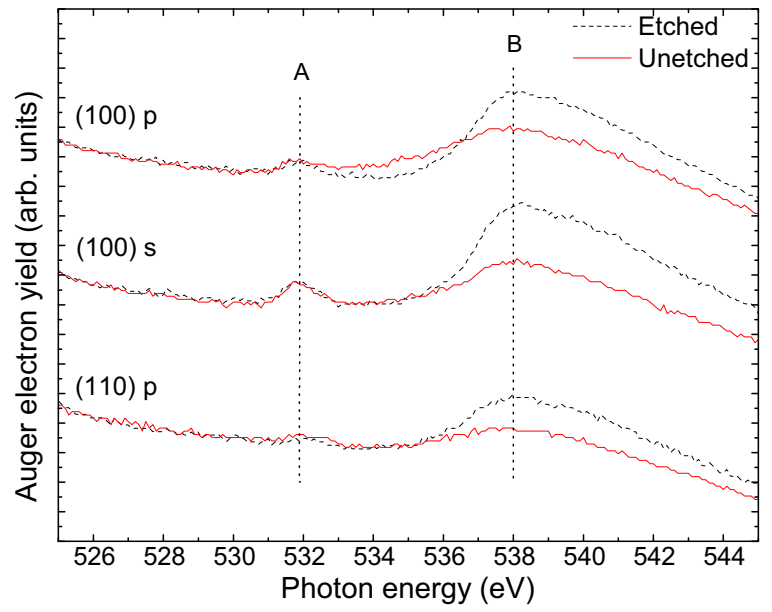

FIG. 5. (Color online) Normalized oxygen $K$-edge AEY NEXAFS for (100) with $p$ and $s$ polarized x-ray beam, and (110) with $p$ polarized beam. Two features labeled $\mathrm{A}$ and $\mathrm{B}$ are observed at $531.9 \mathrm{eV}$ and $538.0 \mathrm{eV}$, respectively.

should thus be associated with all oxygen species on the surface. In each case there is a significant increase in B upon etching, while A remains unchanged. The increase in B upon etching is consistent with a $\sigma^{*}$ resonance, as it agrees with the increased total oxygen on the etched surfaces observed in the $\mathrm{O}(1 s)$ XPS results (Table II).

The significance of peak $\mathrm{A}$ is more ambiguous. Reinke et al. [33] attributed a similar feature to the $\pi^{*}$ resonance associated with ketone groups. Gao et al. [34] also observed such a feature and were unable to determine its origin, as XPS and HREELS measurements of their surface indicated a majority of hydroxyl groups, and were unable to confirm the presence of ketone groups. The populations of double bonded oxygen reported in this work were found by XPS to vary between etched and unetched surfaces, and yet A is not observed to change between these surfaces, as would be expected of a $\pi^{*}$ resonance associated with ketone. This feature does however show a considerable increase in area when analyzed with $s$ polarization compared to $p$ polarization, consistent with species oriented normal to the surface, such as ketone groups. Due to the inconsistencies amongst the literature and our own results, we are unable to determine the origin of A, and cannot explain its apparent stability between the etched and unetched surfaces.
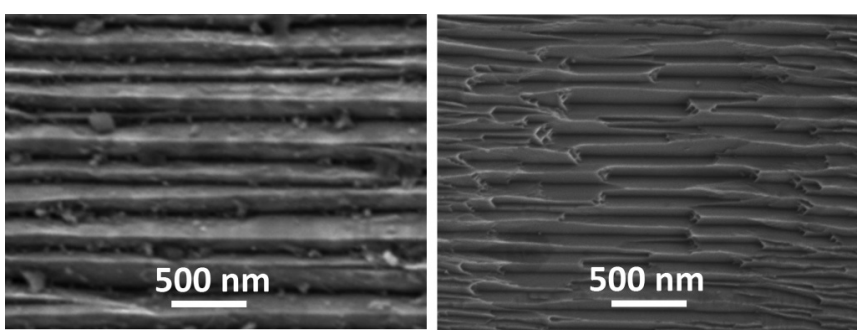

FIG. 6. SEM images of nanostructures produced by etching of (a) (100) and (b) (110) surfaces to depths of 550 and $350 \mathrm{~nm}$, respectively. The polarization of the etching laser was vertical in each case, parallel to [110] in (a) and parallel to [001] in (b).

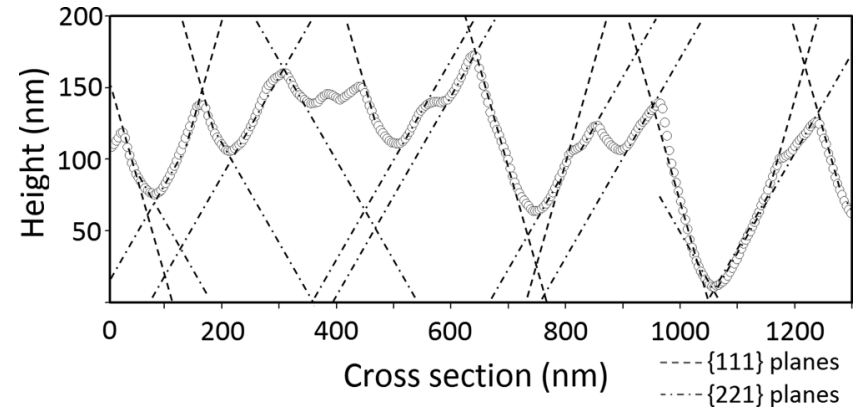

FIG. 7. AFM cross section of the etched (100) surface of Fig. 6(a). Lines indicate sections that fall closely within $\{111\}$ and $\{221\}$ planes.

\section{E. SEM and AFM microscopy}

The XPS and NEXAFS results point to a marked change in chemical environment upon surface etching. Scanning electron microscopy (SEM) and atomic force microscopy (AFM) were used to investigate how such changes are reflected in the morphology of the etched (100) and (110) surfaces. In order to clearly reveal structures that may result from etching, images were recorded for relatively deep etched regions (depths of several hundred nanometers), taking care in each case to ensure that the same beam polarization orientation with respect to the crystal lattice was used as in the preparation of the samples for $\mathrm{x}$-ray analysis. Figure 6 shows the formation of ridges perpendicular to the polarization of the etching beam in each case. The ridge directions are parallel to the intersection of $\{111\}$ planes in the underlying lattice: [011] and [011] in the case of a (100) surface, and [1 10$]$ in the case of a (110) surface. Cross sections of the scanning probe microscopy images show that the faces of the ridges are primarily composed of facets of orientation close to $\{111\}$ and $\{211\}$, as shown for the (100) surface in Fig. 7. The ridges on both types of surface exhibit long range order extending over at least the image size and for more than several microns.

\section{DISCUSSION}

\section{A. (100) surface}

Our characterization of the unetched surface reveals surface termination groups including $s p^{2}, \mathrm{C}-\mathrm{O}, \mathrm{C}=\mathrm{O}$, and possibly $\mathrm{C}-\mathrm{OH}$. Since the as-supplied sample was mechanically polished to $1 \mathrm{~nm}$ roughness, the nominally (100) surface cannot be assumed to be atomically flat but is more likely to be a mixture of microfacets and step edges supporting a multitude of species. There is also likely to be significant lattice damage just below the surface [35] that may contribute to the measured signals. The resulting mixture of surface environments is consistent with the XPS measurements which show approximately equal amounts of singly and doubly bonded oxygen species on the unetched (100) surface.

Upon etching, the conversion of the surface to one dominated by singly bonded oxygen with a larger total oxygen signal is primarily attributed to the formation of a surface composed of $\{111\}$-like microfacets. Facet formation is also supported by comparison with C(1s) XPS measurements of (100) and (111) surfaces made by Takeuchi et al. [18]. In 
their case the (100) surface was wet oxidized in a mixture of $\mathrm{H}_{2} \mathrm{SO}_{4} / \mathrm{HNO}_{3}$ and annealed in argon, whereas in our case it was wet oxidized in $\mathrm{H}_{2} \mathrm{SO}_{4} / \mathrm{H}_{2} \mathrm{O}_{2}$ and annealed in vacuum. Their observed (100) spectrum shows equal areas of singly and doubly bonded oxygen groups, while their (111) spectrum shows a suppressed doubly bonded oxygen peak. Indeed, their (100) and (111) spectra closely correspond to our unetched and etched results of Fig. 2. We conclude that the etched (100) surface develops $\{111\}$-like facets even at the etch depth of less than $10 \mathrm{~nm}$ (roughly 100 atomic layers). The SEM and AFM images confirm that at greater depths the etching induces easily identifiable $\{111\}$-like facets with good long range order.

The total integrated $\mathrm{O}(1 s)$ areas show that there is a higher amount of oxygen detected on the etched surfaces compared to unetched [by $32 \%$ for (100)]. The oxygen signal is likely to be influenced by surface area, oxygen coverage, and possibly also changes in surface texture. In the case of $\{111\}$ facets, the angle relative to the (100) plane is $54.7^{\circ}$ with a 73\% larger surface area. Considering the reduced amount of oxygen supported by fully saturated (111) surfaces compared to (100) (by 33\%), the observed increase in oxygen signal is approximately accounted for by the product of these effects. We note, however, that the C(1s) XPS measurements show approximately equal total oxygen signals for unetched and etched surfaces. Quantitative comparison of absolute signals may be problematic in the present case where there is surface roughening, particularly for the $\mathrm{C}(1 s)$ photoelectrons which originate further into the surface.

\section{B. (110) surface}

The as-supplied (110) surface can be assumed to contain a mixture of (110) and $\{111\}$ facets and a large number of step edges [22]. The unetched surface is populated primarily by singly bonded oxygen groups; a characteristic which is shared with the ether dominated (111) surface. The small changes we observe in the populations of each oxygen species upon etching (less than 30\%) are thus consistent with etch-induced $\{111\}$-like faceting in a similar fashion to that seen for the (100) surface. We note that the fraction of doubly bonded oxygen is not as small on the etched (110) surface as for (100). This may be due to the shallower depth of the (110) etched region resulting in $\{111\}$-like facets which are smaller or less well formed, both of which would cause a larger surface density of step edges.

Etching leads to a notable increase in oxygen signal (by $24 \%$ ) that we attribute primarily to the induced nanostructuring. Oxygen coverage of fully saturated (110) and $\{111\}$ surfaces are equal and this is therefore not considered to be a major factor. The angle of inclination between the $\{111\}$ facets with the surface plane is $35.3^{\circ}$ for $(110)$, so that the increase in surface area for the ideal $\{111\}$ faceted surface over the flat (110) surface is $22 \%$, in close agreement with the observed $\mathrm{O}(1 s)$ XPS signal increase of $24 \%$.

\section{Implications for the etching mechanism}

The surface analysis presented reveals that removal of surface carbon atoms from diamond is accompanied by the development of nanoscale structures that have a form that

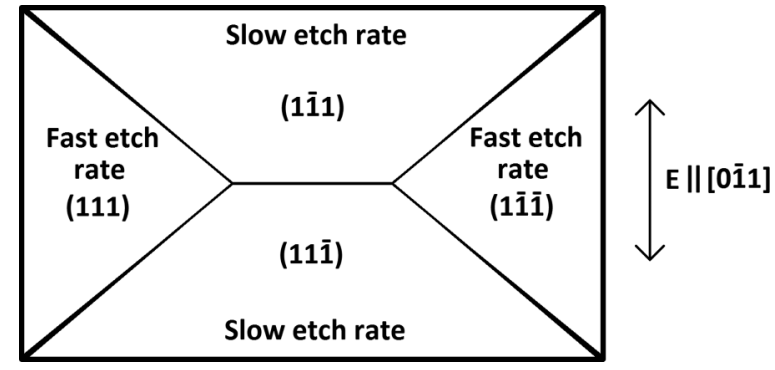

FIG. 8. Simplified diagram of an etched pit bounded by $\{111\}$ facets. The polarization direction of the etching beam is also shown.

is influenced by the directions of crystal lattice planes and writing beam polarization. Since it is reasonable to assume that near room temperature the tightly bound carbon atoms do not migrate across the surface during the etching process, we deduce that structures emerge as material is removed across the whole surface and with a spatially dependent probability sufficiently large to produce structures of peak-to-peak height approximately $20 \%$ of the average depth.

In order to better understand how structures might develop, we consider the case of an etched (100) surface. Our results show that material is removed in a manner that reveals particular underlying $\{111\}$ facets. The resulting extended ridges that form indicate there is a differential in etch rate for facets of a particular orientation with respect to beam polarization. Figure 8 shows the relevant directions in such a case with respect to the laser polarization. As the ridges form perpendicularly to the laser polarization, we deduce that a higher etch rate is experienced by the (111) and (1 $1 \overline{1})$ facets than on the $(1 \overline{1} 1)$ and $(11 \overline{1})$ facets, for which the polarization lies in plane. It is unlikely that polarization information can be preserved for a mechanism involving UV absorption in the bulk followed by the diffusion of energy to the surface. Thus the observed differential etch rate points to a direct interaction of the surface with the light field. We suggest two mechanisms to explain this polarization dependence on etch rate as follows:

(1) Enhanced desorption occurs at step edges of certain orientations with respect to the laser polarization. The desorption of carbon species at such a step edge cause it to propagate across the surface as removal progresses. The polarization dependence causes some facets to experience a higher average etch rate.

(2) Desorption occurs with a probability dependent on the overlap of surface bond directions with the laser polarization. Structures develop as a result of a differential etch rate for facets according to the overlap of the polarization with average carbon-carbon or carbon-oxygen bond directions.

It is presently unclear whether $\mathrm{C}-\mathrm{C}$ bond breakage and desorption is a direct result of absorption by intrinsic surface states, or is the result of some cooperative process involving surface oxygen species or radical sites. A better understanding of how photons couple to the localized surface states is required to test the validity of the above suggested mechanisms. The details of the mechanism will have important implications for the potential smoothness and resolution of structures that can be created using this etching technique. 
At first glance, the etched nanostructures may be seen as similar to the laser induced periodic structures often observed in ablation of materials when using laser intensities close to the ablation threshold [36,37]. These ablated structures are described as wavelike or rippled in directions perpendicular to the laser polarization and have been explained through interference of the laser beam with surface wave excitations [38]. In our case, we discount the possibility for collective surface wave excitations since we observe that the patterns in Fig. 6 are insensitive to the incident laser intensity, provided it remains below the ablation threshold. We have also recently found that the orientation and morphology of the nanostructures changes substantially when using polarization directions other than those investigated here. Such dependence of nanostructuring on the laser polarization with respect to the crystal axes is consistent with the above suggested mechanisms that involve photon coupling with localized and directional bonding groups. A more detailed study of polarization dependence is the subject of a recent report [39].

\section{CONCLUSION}

X-ray surface analysis has revealed that the annealed, wetoxidized, unetched (100) and (110) surfaces were primarily populated by singly bonded oxygen species, most likely ether groups, with a lower, but still significant population of doubly bonded ketone groups. Regions of the samples etched via twophoton UV desorption have a reduced doubly bonded oxygen (ketone) population. The populations of oxygen groups were found to resemble oxidized (111) surfaces, indicating that the surface forms well defined $\{111\}$-like facets very early in the etching process. Such faceting is revealed by AFM and SEM images on more deeply etched samples. Differential etch rates based on the orientation of surface features with respect to the etching laser polarization are proposed as an explanation for the formation of the extended ridge nanostructures.

\section{ACKNOWLEDGMENTS}

The authors are grateful for the assistance of Benjamin Johnston and Peter Ha of the OptoFab, Australian National Fabrication Facility, Debra Birch of the Science Faculty Microscopy Unit, and Adam Joyce for his expertise in optical profilometry. The spectroscopic measurements presented in this research were undertaken on the Soft X-ray Spectroscopy beamline at the Australian Synchrotron, Victoria, Australia. We thank beamline staff Bruce Cowie, Lars Thomsen, and Anton Tadich for their expertise and assistance. This material is based on research sponsored by the Australian Research Council Future Fellowship (FT0990622) and Discovery Grant (DP130103799) Schemes, and the US Air Force Research Laboratory under Agreement No. FA2386-12-1-4055.
[1] P. W. May, Science 319, 1490 (2008).

[2] J. Isberg, J. Hammersberg, D. J. Twitchen, and A. J. Whitehead, Diam. Relat. Mater. 13, 320 (2004).

[3] R. S. Sussman, J. R. Brandon, S. E. Coe, C. S. J. Pickles, C. G. Sweeney, A. Wasenczuk, C. J. H. Wort, and C. N. Dodge, Ind. Diamond Rev. 58, 69 (1998).

[4] S. Prawer and A. D. Greentree, Science 320, 1601 (2008).

[5] J. R. Maze, P. L. Stanwix, J. S. Hodges, S. Hong, J. M. Taylor, P. Cappellaro, L. Jiang, M. V. Gurudev Dutt, E. Togan, A. S. Zibrov, A. Yacoby, R. L. Walsworth, and M. D. Lukin, Nature (London) 455, 644 (2008).

[6] M. J. Burek, N. P. de Leon, B. J. Shields, B. J. M. Hausmann, Y. Chu, Q. Quan, A. S. Zibrov, H. Park, M. D. Lukin, and M. Lončar, Nano Lett. 12, 6084 (2012).

[7] R. P. Mildren, J. E. Downes, J. D. Brown, B. F. Johnston, E. Granados, D. J. Spence, A. Lehmann, L. Weston, and A. Bramble, Opt. Mater. Express 1, 576 (2011).

[8] V. V. Kononenko, M. S. Komlenok, S. M. Pimenov, and V. I. Konov, Quantum Electron. 37, 1043 (2007).

[9] E. Granados, D. J. Spence, and R. P. Mildren, Opt. Express 19, 10857 (2011).

[10] J. Zheng, X. Xie, A. Wee, and K. P. Loh, Diam. Relat. Mater. 10, 500 (2001).

[11] A. Laikhtman and A. Hoffman, Surf. Sci. 522, L1 (2003).

[12] A. Stacey, B. Cowie, J. Orwa, S. Prawer, and A. Hoffman, Phys. Rev. B 82, 125427 (2010).

[13] D. Rebuli, T. Derry, E. Sideras-Haddad, B. Doyle, R. Maclear, S. Connell, and J. Sellschop, Diam. Relat. Mater. 8, 1620 (1999).

[14] N. W. Makau and T. E. Derry, Surf. Rev. Lett. 10, 295 (2003).
[15] T. E. Derry, N. W. Makau, and C. Stampfl, J. Phys.: Condens. Matter 22, 265007 (2010).

[16] P. E. Pehrsson and T. W. Mercer, Surf. Sci. 460, 74 (2000).

[17] P. E. Pehrsson and T. W. Mercer, Surf. Sci. 460, 49 (2000).

[18] D. Takeuchi, S.-G. Ri, N. Tokuda, and S. Yamasaki, Diam. Relat. Mater. 18, 206 (2009).

[19] S. Skokov, B. Weiner, and M. Frenklach, Phys. Rev. B 55, 1895 (1997).

[20] K. Bobrov, H. Shechter, A. Hoffman, and M. Folman, Appl. Surf. Sci. 196, 173 (2002).

[21] B. L. Mackey, J. N. Russell, Jr., J. E. Crowell, P. E. Pehrsson, B. D. Thoms, and J. E. Butler, J. Phys. Chem. B 105, 3803 (2001).

[22] T. W. Mercer, J. N. Russell, Jr., and P. E. Pehrsson, Surf. Sci. 392, L21 (1997).

[23] R. Klauser, J.-M. Chen, T. Chuang, L. Chen, M. Shih, and J.-C. Lin, Surf. Sci. 356, L410 (1996).

[24] J. Wilson, J. Walton, and G. Beamson, J. Electron Spectrosc. Relat. Phenom. 121, 183 (2001).

[25] G. Speranza, S. Torrengo, A. Miotello, L. Minati, I. Bernagozzi, M. Ferrari, M. Dipalo, and E. Kohn, Diam. Relat. Mater. 20, 560 (2011).

[26] M. P. Seah and W. A. Dench, Surf. Interface Anal. 1, 2 (1979).

[27] J. Stöhr and D. A. Outka, Phys. Rev. B 36, 7891 (1987).

[28] C. Monachon and L. Weber, J. Appl. Phys. 113, 183504 (2013).

[29] P. Mérel, M. Tabbal, M. Chaker, S. Moisa, and J. Margot, Appl. Surf. Sci. 136, 105 (1998).

[30] L. Diederich, O. Küttel, P. Ruffieux, T. Pillo, P. Aebi, and L. Schlapbach, Surf. Sci. 417, 41 (1998). 
[31] L. S. De Bernardez, J. Ferrón, E. C. Goldberg, and R. H. Buitrago, Surf. Sci. 139, 541 (1984).

[32] A. Laikhtman, I. Gouzman, A. Hoffman, G. Comtet, L. Hellner, and G. Dujardin, J. Appl. Phys. 86, 4192 (1999).

[33] P. Reinke, A. Knop-Gericke, M. Hävecker, and T. SchedelNiedrig, Surf. Sci. 447, 229 (2000).

[34] X. Gao, L. Liu, D. Qi, S. Chen, A. Wee, T. Ouyang, K. Loh, X. Yu, and H. Moser, J. Phys. Chem. C 112, 2487 (2008).
[35] J. R. Hird, in Optical Engineering of Diamond, edited by R. P. Mildren and J. R. Rabeau (Wiley-VCH, New York, 2013), Chap. 3, pp. 71-107.

[36] M. Birnbaum, J. Appl. Phys. 36, 3688 (1965).

[37] J. F. Young, J. S. Preston, H. M. van Driel, and J. E. Sipe, Phys. Rev. B 27, 1155 (1983).

[38] J. E. Sipe, J. F. Young, J. S. Preston, and H. M. van Driel, Phys. Rev. B 27, 1141 (1983).

[39] A. Lehmann, C. Bradac, and R. P. Mildren, Nat. Commun. 5, 3341 (2014). 